Original Research

\title{
Evaluation of the Prevalence of Screening for Dysphagia among Older People Admitted to Medical Services - An International Survey
}

David Smithard $^{1,+}$, Signe Westmark ${ }^{2,+}$, Dorte Melgaard ${ }^{2,3,+, *}$

1. Queen Elizabeth Hospital, Stadium Road, Woolwich, London SE18 4QH, United Kingdom; EMail: david.smithard@nhs.net

2. Centre for Clinical Research, Bispensgade 37, 9800 Hjørring, Denmark; E-Mails: s.westmark@rn.dk; dmk@rn.dk

3. Department of Clinical Medicine, Aalborg University, Aalborg, Denmark

† These authors contributed equally to this work.

* Correspondence: Dorte Melgaard; E-Mail: $\underline{\mathrm{dmk} @ r n . d k}$

Academic Editor: James S. Powers

Special Issue: Dysphagia in the Elderly

\section{OBM Geriatrics}

2019 , volume 3 , issue 4

doi:10.21926/obm.geriatr.1904086
Received: September 15, 2019

Accepted: November 08, 2019

Published: November 15, 2019

\begin{abstract}
Background: With the increasing age of the world population, the number of old and frail people is increasing. Respiratory disease is a common reason for hospital admission for older people. Aspiration of saliva, which is probably infected, is the likely etiological agent. The incidence of an abnormal swallow is high in frail older people. Despite the high incidence of swallow problems among older people, many hospital services do not routinely screen older people for dysphagia.
\end{abstract}

Methods: A survey, using Research Electronic Database Capture (REDCap), was carried out via email for a convenience sample of contacts working in hospitals worldwide. They were asked; 1 ) type of medical services, 2 ) does your service screen older (>80 years) people for swallowing problems, 3 ) have nursing staff and medical staff trained to administer a swallow

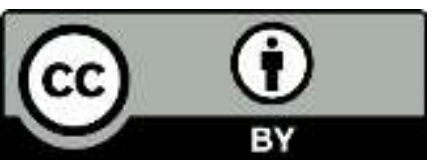

(C) 2019 by the author. This is an open access article distributed under the conditions of the Creative Commons by Attribution License, which permits unrestricted use, distribution, and reproduction in any medium or format, provided the original work is correctly cited. 
screen, 4) is a swallowing rehabilitation program offered to older people, and 5) which elements of a rehabilitation program are offered and used.

Results: One hundred and fifty people with consent from 29 countries responded to the survey. Of them, $75 \%$ work in an acute hospital, $15 \%$ in rehab, and $10 \%$ in community. In total, $62 \%$ responded that they do not or only occasionally screen older people for swallowing difficulties; $50 \%$ of the medical staff and $27 \%$ of the nursing staff were not trained to administer a swallowing screen. A rehabilitation swallowing program was offered in $63 \%$ cases, with chin tuck against resistance (83\%), tongue strengthening $(87 \%)$, and shaker maneuver (79\%) being more common than electric stimulation (21\%).

Conclusions: The results of this study suggest that most health facilities do not routinely screen older people for swallowing problems, and the majority of them do not train their staff. The majority of facilities were offering a rehabilitation program. In addition, it is likely that many people are not receiving the appropriate proactive intervention because swallowing problems are not being proactively sought.

\section{Keywords}

Dysphagia; screening; swallowing disorders; elderly; hospital; prevalence; survey; rehabilitation; medical services

\section{Introduction}

In the last few decades, the average age of humans has drastically increased. In 2019, the European population was $513,480,691$, of which $5.67 \%(29,114,355)$ were $>80$ years of age [1]. With advanced age, people accumulate long term conditions and become increasingly frail, $24 \%$ being frail by the age of 85 years [2]. The combination of frailty and multiple comorbidities results in increased use of healthcare resources including admissions to hospitals [2].

The incidence of pneumonia in Europe for those $>75$ years is up to $65.2 \%$ for men and $19.6 \%$ for women. Respiratory infection is a common reason for admission to hospital. Admission to hospitals due to pneumonia used 49.63 million bed days in Europe (length of stay 5-13 days) [1]. Mortality secondary to pneumonia in those with an age of $>80$ years was 25/100 000 and total cost in the region was 10.1 billion Euros [3]. In about 5\%-18\% cases, community-acquired pneumonia is secondary to aspiration [4, 5]. In 2017, 940,341 people were admitted to hospital in England during 2018 with pneumonia, 44.45\% $(417,984)$ were of 80 years or older [6]. A further 61,248 people were admitted with aspiration pneumonitis, $93 \%(57,223)$ of them were over the age of 80 years.

The mechanics of swallowing changes with age, for some, this has no effect as they subtly and subconsciously change their diet (food consistency and volume) and way of swallowing (smaller mouthfuls, double swallows) with many not reporting any difficulties to their family or doctor [7, 8]. Studies have suggested that $13 \%$ of older people living in the community have problems of swallowing, which goes up to $100 \%$ in people with severe progressive neurological disease (Parkinson's disease, Motor neuron disease) [9]. 
Older people with multiple comorbidities including frailty are more prone to developing a chest infection than robust older people, due to the suppression of their immunity and the ongoing inflammatory response associated with their underlying medical problems [10]. The literature documents that swallowing problems or dysphagia are linked to unrecognized malnutrition, which is highly relevant in the hospitals $[11,12]$.

Dysphagia is recognized as a major complication of stroke and as a part of good stroke care, it must be assessed at the time of admission (within $4 \mathrm{~h}$ ). Bray et al. noted that a delay in this assessment increases the risk of pneumonia [13]. Pneumonia is a common reason for admission and is often accompanied by dysphagia (either a primary problem or secondary to physiological decompensation). It is not unreasonable to expect all older and frail people to be screened for swallowing problems at the time of admission.

This study was undertaken to understand whether medical services admitting frail older people acutely routinely screen all older people for oropharyngeal dysphagia (OD), whether they have trained their staff to identify OD, and whether swallowing rehabilitation has been offered.

\section{Materials and Methods}

In order to evaluate the prevalence of screening for hospitalized older people, a survey was conducted. The survey consisted of five questions; 1 ) type of medical service, 2 ) does your service screen older (>80 years) people for swallowing problems, 3 ) are nursing staff and medical staff trained to administer a swallow screen, 4) is a swallowing rehabilitation program offered to older people, and 5) which rehabilitation program is offered and used. The survey was emailed to a convenience sample of contacts working in healthcare settings/services across the world, the contacts were encouraged to forward the survey to other relevant people in their network. Only one response was requested per service. Data were collected and managed using Research Electronic Database Capture (REDCap) tools hosted at North Denmark Region [14, 15]. REDCap is a secure, web-based software platform designed to support data capture for research studies.

The advice was sought from the Local Ethics Committee and formal ethics approval was not required. In the questionnaire, the participants confirmed their consent for the results of the survey to be published.

\section{Results}

In total, 155 people from 29 countries responded and completed the survey; however, five did not consent to publish data, thus they were excluded from further analysis. Owing to the limitation of the employed method, the response rate could not be determined. Of the 150 respondents, $75 \%$ work in an acute hospital, $15 \%$ in rehabilitation settings, and $10 \%$ in the community (Figure 1).

The majority of health services $(93 / 150(62 \%))$ do not or only occasionally screen older people for swallowing difficulties (Figure 2).

In order to offer a screening service, trained staff is required, our results show that $50 \%$ of the medical staff and $27 \%$ of the nursing staff were not trained to administer a swallowing screen (Figure 3 and Figure4).

A swallowing rehabilitation program was offered by $63 \%$ of services (Figure 5). 
Within these programs, chin tuck against resistance (83\%), tongue strengthening (87\%), Shaker maneuver (79\%), and transcutaneous electric stimulation (21\%), were provided (Figure 6).

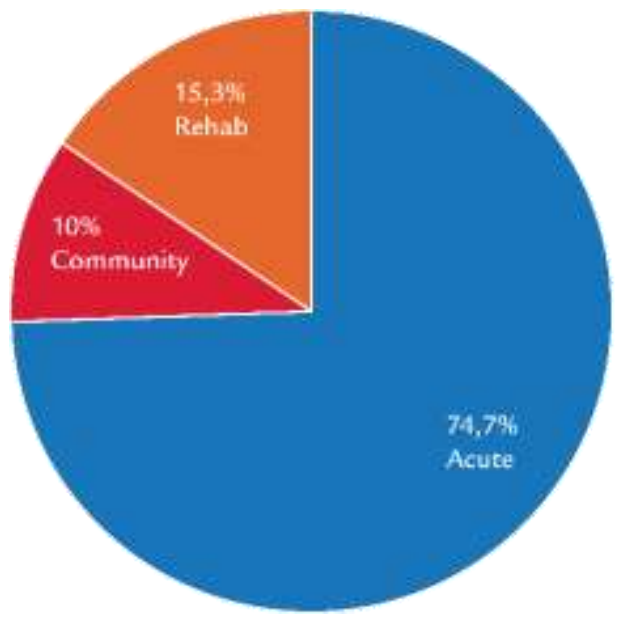

Figure 1 Type of Service

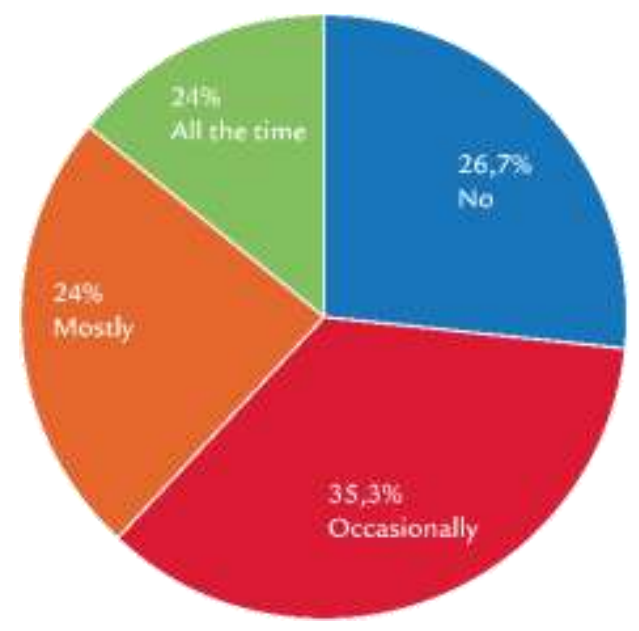

Figure 2 Does your hospital screen older people?

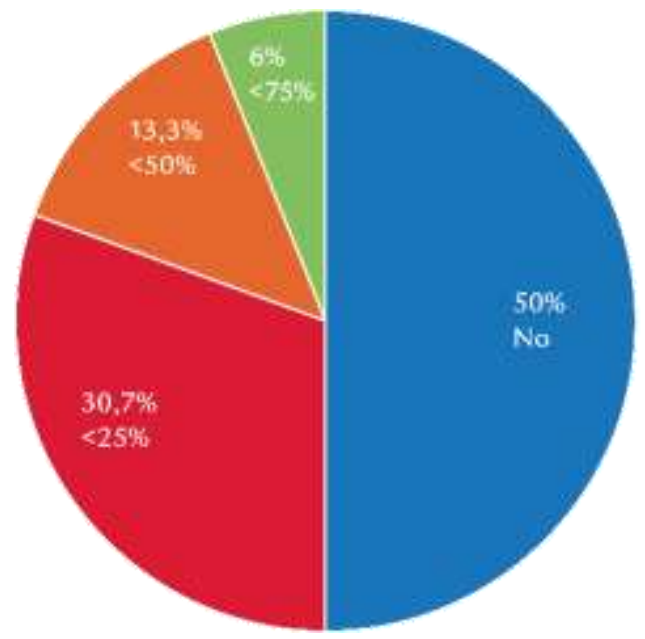

Figure 3 Are the medical staff trained to administer a swallow screen? 


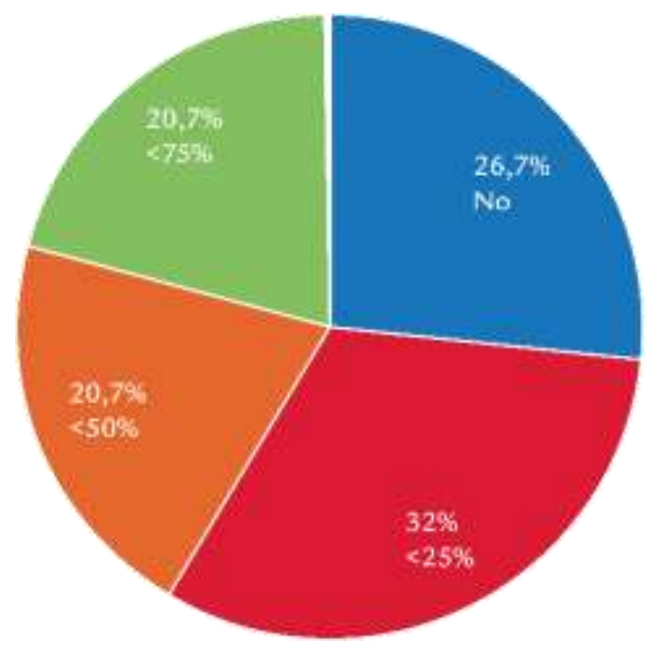

Figure 4 Are the nursing staff trained to administer a swallow screen?

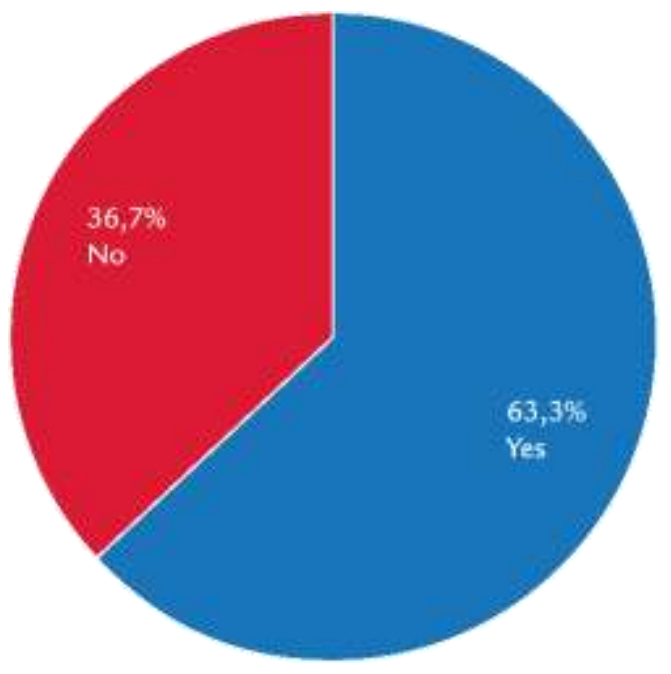

Figure 5 Is a swallowing rehabilitation program offered to older people?

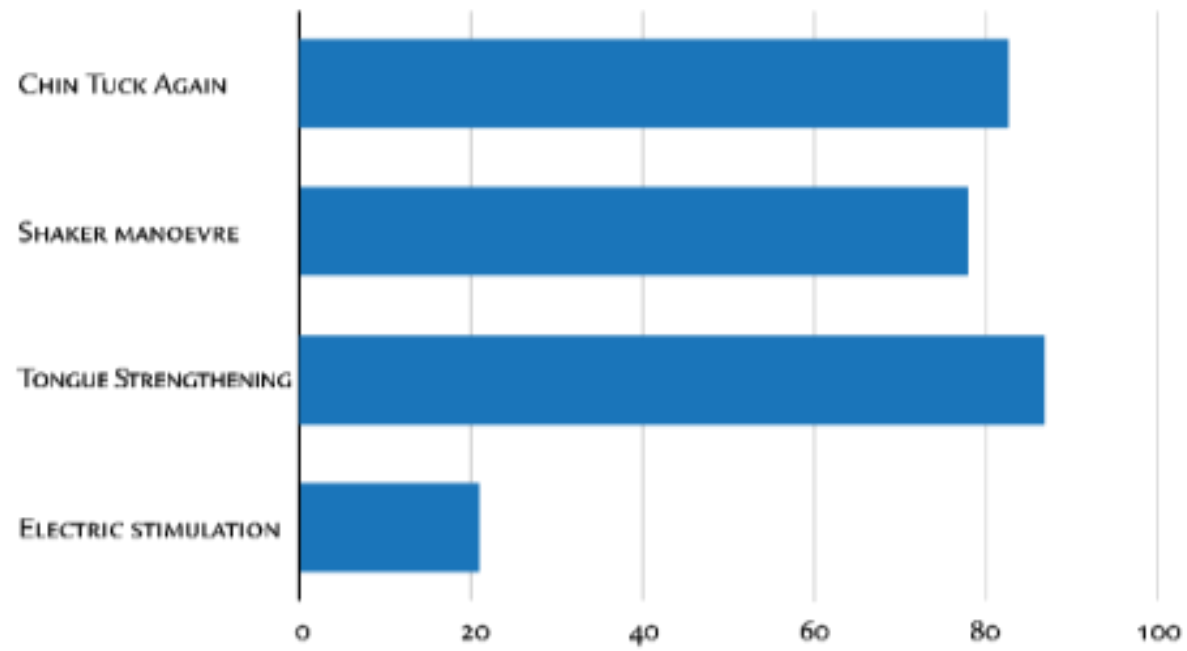

Figure 6 Which rehabilitation interventions are offered? 


\section{Discussion}

The results of this study have shown that the majority of hospitals do not routinely screen older people for the presence of dysphagia. It is well documented that the prevalence of dysphagia is high in older people and especially if they are hospitalized or dwelling in a nursing home [11, 1618]. In recent years, several international and local guidelines have been suggested indicating that it is a good practice to screen for OD in patients with acute stroke [19]. Despite the prevalence of dysphagia in older people, particularly those admitted to the hospital and are comparable to the patients with prevalent dysphagia in acute stroke, as documented in the present study, there is no routine practice to screen older people when they are hospitalized or admitted to medical services. No study has documented the effect of early or routine screening among older people.

Dysphagia is a geriatric syndrome. The failure to identify early, any swallowing problems in older adults, will increase the likelihood of protein-calorie malnutrition and pneumonia resulting in increased morbidity, length of stay, admission to nursing homes and mortality. The conclusion to be drawn is that it is appropriate to screen for the presence of OD at the time of admission [9]. Early identification will enable healthcare staff to intervene and potentially improve nutrition, muscle strength and outcome [9].

Rehabilitation is individualized and no particular rehabilitation strategy applies to all older patients with OD, and the evidence for the effect of compensatory strategies for OD is low [9]. The presence of weak evidence in earlier studies has suggested that compensatory swallowing strategies and exercises are difficult for many older people [9, 20]. However, some clinical practices support their use as older people have shown good results and improved swallowing using these strategies. Considering this, it is surprising that there, as illustrated in the present study, is still a relatively large portion that is not trained to apply these strategies and in the same way, it is not offered in many medical or rehabilitation services.

As the number of older people is increasing, the potential cost to health economy is increasing. Screening older people for the presence of $O D$ on admission by the medical or rehabilitation service has the potential to reduce morbidity and mortality. Further research is required to identify appropriate and simple screening tools and analyze the effect of screening on outcome. In the same way, there is a need for more high evidential studies documenting the effect of compensatory rehabilitation strategies.

The limitations of the present study are due to the use of personal and professional contacts for the completion of the survey. As we sent the survey to a number of people and encouraged them to disseminate it further to their colleagues, we do not know the percentage of respondents compared to those contacted. We focused only on acute medical services and the conclusions about other aspects of healthcare (community and rehabilitation) cannot be reliably drawn.

\section{Conclusions}

The existing literature states that older people admitted acutely to hospital with OD have a poor outcome. Despite this observation, this study documents that it is still not a common practice to screen for OD at the admission of the older people to hospitals or other healthcare settings nor to offer compensatory strategies during the hospitalization. Further research is needed to uncover the effect of early identification of OD. In the meantime, guidelines should recommend screening for the presence of OD in older people admitted to healthcare facilities. 


\section{Acknowledgments}

The authors thank all participating institutions and participants for complete the questionnaire.

\section{Author Contributions}

All the Authors participated in the concept, design, analysis, writing and revision of the manuscript.

\section{Competing Interests}

The authors have declared that no competing interests exist.

\section{References}

1. World Helth Orgnization. Eurostats European Health Information Gateway European Mortality Database. Available from: https://gateway.euro.who.int/en/datasets/european-mortalitydatabase/\#population-and-icd-used.

2. Xue QL. The frailty syndrome: Definition and natural history. Clin Geriatr Med. 2011; 27: 1-15.

3. Welte T, Torres A, Nathwani D. Clinical and economic burden of community-acquired pneumonia among adults in Europe. Thorax. 2012; 67: 71-79.

4. Sanivarapu RR, Gibson JG. Aspiration pneumonia. StatPearls. 2019.

5. Komiya K, Ishii H, Kadota J. Healthcare-associated pneumonia and aspiration pneumonia. Aging Dis. 2015; 6: 27-37.

6. NHS Digital. Hospital admitted patient care activity, 2017-2018. Available from: https://digital.nhs.uk/data-and-information/publications/statistical/hospital-admittedpatient-care-activity.

7. Omari TI, Kritas S, Cock C, Besanko L, Burgstad C, Thompson A, et al. Swallowing dysfunction in healthy older people using pharyngeal pressure-flow analysis. Neurogastroenterol Motil. 2014; 26: 59-68.

8. Chen PH, Golub JS, Hapner ER, Johns MM, 3rd. Prevalence of perceived dysphagia and qualityof-life impairment in a geriatric population. Dysphagia. 2009; 24: 1-6.

9. Baijens LW, Clave P, Cras P, Ekberg O, Forster A, Kolb GF, et al. European society for swallowing disorders- European Union Geriatric Medicine Society white paper: Oropharyngeal dysphagia as a geriatric syndrome. Clin Interv Aging. 2016; 11: 1403-1428.

10. Li H, Manwani B, Leng SX. Frailty, inflammation, and immunity. Aging Dis. 2011; 2: 466.

11. Carrion S, Cabre M, Monteis R, Roca M, Palomera E, Serra-Prat M, et al. Oropharyngeal dysphagia is a prevalent risk factor for malnutrition in a cohort of older patients admitted with an acute disease to a general hospital. Clin Nutr. 2015; 34: 436-442.

12. Namasivayam AM, Steele CM. Malnutrition and dysphagia in long-term care: A systematic review. J Nutr Gerontol Geriatr. 2015; 34: 1-21.

13. Bray BD, Smith CJ, Cloud GC, Enderby P, James M, Paley L, et al. The association between delays in screening for and assessing dysphagia after acute stroke, and the risk of strokeassociated pneumonia. J Neurol Neurosurg Psychiatry. 2017; 88: 25-30. 
14. Harris PA, Taylor R, Thielke R, Payne J, Gonzalez N, Conde JG. Research electronic data capture (REDCap)--a metadata-driven methodology and workflow process for providing translational research informatics support. J Biomed Inform. 2009; 42: 377-381.

15. Harris PA, Taylor R, Minor BL, Elliott V, Fernandez M, O'Neal L, et al. The REDCap consortium: Building an international community of software platform partners. J Biomed Inform. 2019; 95: 103208.

16. Cabré M, Almirall J, Clave P. Aspiration pneumonia: Management in Spain. Eur Geriatr Med. 2011; 2: 180-183.

17. Altman KW, Yu GP, Schaefer SD. Consequence of dysphagia in the hospitalized patient: Impact on prognosis and hospital resources. Arch Otolaryngol Head Neck Surg. 2010; 136: 784-789.

18. Melgaard D, Rodrigo-Domingo M, Morch MM. The Prevalence of oropharyngeal dysphagia in acute geriatric patients. Geriatrics. 2018; 3: E15.

19. Smith EE, Kent DM, Bulsara KR, Leung LY, Lichtman JH, Reeves MJ, et al. Effect of dysphagia screening strategies on clinical outcomes after stroke: a systematic review for the 2018 guidelines for the early management of patients with acute ischemic stroke. Stroke. 2018; 49: e123-e128.

20. Ney DM, Weiss JM, Kind AJ, Robbins J. Senescent swallowing: Impact, strategies, and interventions. Nutr Clin Pract. 2009; 24: 395-413.

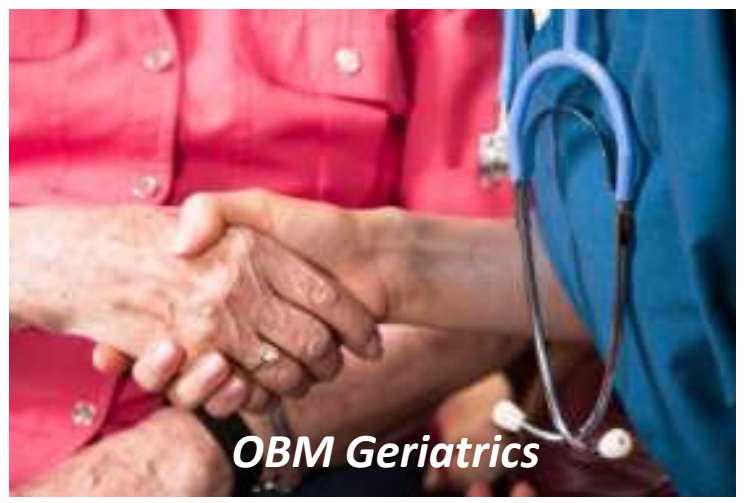

Enjoy OBM Geriatrics by:

1. Submitting a manuscript

2. Joining in volunteer reviewer bank

3. Joining Editorial Board

4. Guest editing a special issue

For more details, please visit:

http://www.lidsen.com/journals/geriatrics 